\title{
Computation of Faber Series With Application to Numerical Polynomial Approximation in the Complex Plane
}

\author{
By S. W. Ellacott*
}

\begin{abstract}
Kövari and Pommerenke [19], and Elliott [8], have shown that the truncated Faber series gives a polynomial approximation which (for practical values of the degree of the polynomial) is very close to the best approximation. In this paper we discuss efficient Fast Fourier Transform (FFT) and recursive methods for the computation of Faber polynomials, and point out that the FFT method described by Geddes [13], for computing Chebyshev coefficients can be generalized to compute Faber coefficients.

We also give a corrected bound for the norm of the Faber projection (that given in Elliott [8], being unfortunately slightly in error) and very briefly discuss a possible extension of the method to the case when the mapping function, which is required to compute the Faber series, is not known explicitly.
\end{abstract}

1. Introduction. Several algorithms for numerical minimax approximation which are applicable in the complex plane have been proposed (e.g. Ellacott and Williams [6]; Barrodale, Delves and Mason [2]; Blatt [3]; Gutknecht [17]; Opfer [25]; Elliott [8]; Streit and Nuttall [28]; Glashoff and Roleff [14], are some of the most recent). The earlier of these methods seem expensive computationally. Although later ones appear possibly to be more efficient, it is natural to look for expansion methods, analogous to the Chebyshev series for real approximation which give "nearly best" polynomial approximations when the expansion is truncated. The Faber series provides such an expansion (Kövari and Pommerenke [19]; Elliott [8]), and the main purpose of this paper is to discuss the efficient numerical computation of such series. (For a rather different approach to "near best" polynomial approximation, see Trefethen [31]; Gutknecht and Trefethen [18]).

To make the notion of near best approximation more precise, we may employ the ideas of Cheney and Price [4], and Mason [24]; see also Geddes and Mason [12], and Geddes [13], for the application to complex approximation. Let $D$ be a bounded, closed continuum in the complex plane with boundary $\Gamma$ such that the complement of $D$ is simply connected in the extended plane and contains the point at $\infty$. We denote by $A(D)$ the space of functions which are continuous at every point of $D$ and analytic at every interior point. $\hat{A}(D)$ will denote the subspace of functions which are analytic at every point of $D$. Now let $P_{n}$ denote the space of complex polynomials of degree $\leqslant n$. Given $f \in A(D)$, it is well known that there exists a unique best

Received February 4, 1981; revised August 19, 1981 and July 14, 1982.

1980 Mathematics Subject Classification. Primary 65D15; Secondary 30B50, 30E10, 41A10, 4lA50, 41A58, $65 \mathrm{E} 05$.

Key words and phrases. Faber series, near best approximation.

*Author partially supported by a Royal Society European Visiting Fellowship. 
minimax approximation $p_{n}$ to $f$ from $P_{n}$, i.e.

$$
\left\|f-p_{n}\right\|_{\infty} \leqslant\|f-p\|_{\infty} \text { for all } p \in P_{n},
$$

where \|\|$_{\infty}$ denotes the uniform norm, $\|f\|_{\infty}=\max _{z \in D}|f(z)|$.

Suppose we have a projection $C_{n}: A(D) \rightarrow P_{n}$ (i.e. $C_{n}$ is a bounded linear operator which satisfies $\left.C_{n}(p)=p, p \in P_{n}\right)$. We have

$$
f-C_{n}(f)=f-p_{n}+C_{n}\left(p_{n}-f\right)
$$

whence

$$
\left\|f-C_{n}(f)\right\|_{\infty} \leqslant\left(1+\left\|C_{n}\right\|\right)\left\|f-p_{n}\right\|_{\infty} .
$$

Thus if $\left\|C_{n}\right\|$ is reasonably small, $C_{n}(f)$ will be almost as good an approximation to $f$ as the best approximation $p_{n}$ (e.g., if $\left\|C_{n}\right\| \leqslant 9$, we will not lose more than one decimal place accuracy in accepting $C_{n}(f)$ as an approximation to $f$, rather than $p_{n}$ ).

Bounds on the norms of the projections obtained by truncating the Maclaurin expansion on the unit disc, and Chebyshev series on ellipses, were given in Geddes and Mason [12], and Geddes [13], respectively. Elliott [8], pointed out that a natural generalization of these ideas to an arbitrary $D$ is given by the Faber expansion, and that, moreover, the bounds given by Geddes and Mason, and Geddes, are rather similar (although actually somewhat sharper for the special cases considered) to known results on truncated Faber series (Kövari and Pommerenke [19]). We shall give a further discussion of these results in the next section.

2. Definition and Properties of the Faber Series. A general description of the Faber series is given in Markushevich [22] (Chapter 3 of vol. 3). See also Curtiss [5]; and Gaier [11]. For a region** $D$ as defined in Section 1, we have a mapping $\varphi$ which maps the complement of $D$ conformally onto the complement of a closed disc of radius $\rho$ with center at the origin, and which satisfies the condition $\lim _{z \rightarrow \infty} \varphi(z) / z=1$. Here $\rho$ is the so-called logarithmic capacity, or transfinite diameter, of $D$. Let $\psi$ be the inverse of $\varphi$.

The level curves $\Gamma_{R}(R>\rho)$ of $D$ are the images under $\psi$ of the circles $|w|=R$, and we denote by $I_{R}$ the closed Jordan region with boundary $\Gamma_{R}$. The $n$th Faber polynomial $\varphi_{n}$ is the polynomial part of the Laurent expansion at $\infty$ of $(\varphi)^{n}$. Thus $\varphi_{0} \equiv 1$ and $\varphi_{n}$ is a monic polynomial of degree $n$.

Given $f \in \hat{A}(D)$, the Faber coefficient $a_{n}$ is defined by

$$
a_{n}=\frac{1}{2 \pi i} \int_{|w|=R} \frac{f(\psi(w))}{w^{n+1}} d w,
$$

where $R>\rho$ is sufficiently small that $f$ can be extended analytically to $I_{R}$. If $\psi$ can be extended continuously to the boundary $|w|=\rho$ (e.g. this is the case if $\Gamma$ is a Jordan curve) ${ }^{* * *}$, then the value $R=\rho$ is also acceptable. The Faber series $\sum_{j=0}^{\infty} a_{j} \varphi_{j}$ converges uniformly and absolutely to $f$ on every $I_{R}$ to which $f$ can be extended

\footnotetext{
${ }^{* *}$ The term region here and elsewhere is convenient for describing $D$. It is not, of course, used in the sense of some authors to denote a specifically open connected set.

${ }^{* * *}$ Here and below, where such an extension is possible, we refer also to the extended function as $\psi$. (The requirement that $\psi$ be continuous, can, of course, be considerably weakened.) Similarly we do not distinguish between $f$ and its analytic extension to $I_{R}$.
} 
analytically. For $f \in A(D)-\hat{A}(D)$, the Faber coefficient can be defined by replacing $R$ by $\rho$ in (2.1) provided the integral exists. (For a discussion of the convergence of the Faber series in this case, see e.g. Kövari and Pommerenke [19].)

The Faber projection $F_{n}$ is, of course, obtained by truncating the series, i.e. $F_{n}(f)=\sum_{j=0}^{n} a_{j} \varphi_{j}$. We require (compare (1.1)) bounds on $\left\|F_{n}\right\|$. It is possible to obtain at least qualitative bounds without further restriction on $D$ (Kövari and Pommerenke [19]), but asymptotically sharper results as $n \rightarrow \infty$ are obtained if we assume that $\Gamma$ is of bounded total rotation; moreover the bounds are then easily made quantitative. If $D$ is a closed Jordan region and $\Gamma$ is rectifiable, there exists at almost every point $z \in \Gamma$ a tangent vector which makes an angle $\theta(z)$ with the positive real axis. $\Gamma$ has (bounded) total rotation $V$ if the quantity

$$
V=\int_{\Gamma}|d \theta(z)|
$$

is finite. Clearly $V \geqslant 2 \pi$ and $V=2 \pi$ if $D$ is convex. More generally if $\Gamma$ is made up of simple arcs, $V$ is often easy to calculate. The results discussed below remain true for the case in which $D$ degenerates to a Jordan arc. In this case all integrals must be interpreted as being along both "sides" of the arc and "round" the ends. Given any fixed point $z_{0} \in \Gamma$, we have (Radon [27])

$$
\int_{\Gamma}\left|d_{z} \arg \left(z-z_{0}\right)\right| \leqslant V
$$

where the subscript $z$ signifies that $\arg \left(z-z_{0}\right)$ is to be considered as a function of $z$, and where the jump in $\arg \left(z-z_{0}\right)$ at $z=z_{0}$ is equal to the exterior angle of $\Gamma$ at $z_{0}$.

We now give several other representations of the Faber polynomials $\varphi_{n}$ which we collect together as a single theorem.

THEOREM 2.1. (a) For a general region $D$ the following representations of $\varphi_{n}$ are valid.

(i) $\varphi_{n}(z)=\sum_{j=0}^{n} c_{j} z^{j}$, where

$$
c_{j}=\frac{1}{2 \pi i} \int_{|z|=R} \frac{\{\varphi(z)\}^{n}}{z^{j+1}} d z
$$

with $R$ chosen sufficiently large so that $D$ is contained in the interior of the region bounded by the circle $|z|=R$. Alternatively,

$$
c_{j}=\frac{1}{2 \pi i} \int_{|w|=s} \frac{w^{n} \psi^{\prime}(w)}{\{\psi(w)\}^{j+1}} d w, \quad s>\rho .
$$

(ii) $\varphi_{n}(z)$ is the coefficient of $w^{-(n+1)}$ in the expansion at $\infty$ of the generating function $\psi^{\prime}(w) /(\psi(w)-z)$.

(iii)

$$
\varphi_{n+1}(z)=z \varphi_{n}(z)-\sum_{k=0}^{n-1} b_{k} \varphi_{n-k}(z)-(1+n) b_{n}, \quad n \geqslant 0,
$$

where $b_{k}$ is the coefficient of $w^{-k}$ in the expansion of $\psi(w)$ at $\infty$. 
(b) If $D$ is a Jordan region whose boundary $\Gamma$ is of bounded total rotation (or for the degenerate case when $D$ is an arc as described above $)$, we have for each $z_{0}=\psi\left(\rho e^{i \theta_{0}}\right)$

$$
\varphi_{n}\left(\psi\left(\rho e^{i \theta}\right)\right)=\frac{\rho^{n}}{\pi} \int_{0}^{2 \pi} e^{i n \theta} d_{\theta} v\left(\theta, \theta_{0}\right), \quad n \geqslant 1,
$$

where $v\left(\theta, \theta_{0}\right)=\arg \left(\psi\left(\rho e^{i \theta}\right)-\psi\left(\rho e^{i \theta_{0}}\right)\right)$.

Proofs. (ai) (2.3) is simply the expression for the Laurent coefficient in the expansion of $(\varphi)^{n}$. (2.4) is obtained by the substitution $z=\psi(w)$.

(aii) See e.g. Markushevich [22].

(aiii) (2.5) is obtained by comparing coefficients in (aii).

It may be found, for example, in the proof of Theorem 1 in Kövari and Pommerenke [19]; or in Curtiss [5]; but its potential usefulness as a computational device does not appear to have been previously considered.

(b) See Pommerenke [26]. The proof there is given only for the case $\rho=1$, but modification for the general case is straightforward.

Theorem 2.1(b) leads to a simple bound on $\left\|\varphi_{n}\right\|_{\infty}$. This can be used to obtain an a priori bound on the error of the truncated series which is, at least in principle, computable.

(For a bound on $\left\|\varphi_{n}\right\|_{\infty}$ when $\Gamma$ is not of bounded total rotation seee Kövari and Pommerenke [19]).

Corollary 2.2. (a) Let $D$ and $\Gamma$ be as in Theorem 2.1(b), and let $V$ be the total rotation of $\Gamma$ (interpreted appropriately in the case when $D$ degenerates to an arc). Then we have for $n \geqslant 1$,

$$
\left\|\varphi_{n}\right\|_{\infty} \leqslant \rho^{n} V / \pi .
$$

This bound is best possible in the sense that when $D \equiv[-1,1]$, equality holds.

(b) If $f \in \hat{A}\left(I_{R}\right), R>\rho$, we have, for $n \geqslant 0$,

$$
\left\|f-F_{n}(f)\right\|_{\infty} \leqslant \frac{M_{r}(R) V}{\pi} \frac{(\rho / R)^{n+1}}{1-\rho / R},
$$

where $M_{r}(R)=\max _{z \in \Gamma_{R}}|f(z)|$.

Proof. (a) In view of the maximum principle, $\left|\varphi_{n}(z)\right|, z \in D$, achieves its maximum value at some $z_{0} \in \Gamma$. The bound then follows from (2.6), noting (2.2).

When $D \equiv[-1,1], V=2 \pi$ and (see e.g. Markushevich [22, p. 106]), $\varphi_{n}=T_{n} / 2^{n-1}$ where $T_{n}$ is the $n$th degree Chebyshev polynomial of the first kind. For this region $D$, $\rho=\frac{1}{2}$ and equality is indeed achieved since $\left\|T_{n}\right\|_{\infty}=1$.

(b) follows straightforwardly from (a) and the bound $\left|a_{n}\right| \leqslant M_{f}(R) / R^{n}$ (see Markushevich [22, vol. 3, p. 109]).

Now we conclude this section by discussing $\left\|F_{n}\right\|$. (Note that since $\hat{A}(D)$ is dense in $A(D)$, it does not matter which space we consider here.)

The result given below is essentially due qualitatively to Kövari and Pommerenke [19], and quantitatively to Elliott [8] (although not actually expressed as a bound on $\left.\left\|F_{n}\right\|\right)$. However, we give part of the proof for two reasons. First, it is not entirely clear in the former paper whether the authors are discussing a general region or one of logarithmic capacity $\rho=1$, and in fact the proof as it stands is only correct for this latter special case. (It so happens, however, that the effect of a different $\rho$ 
cancels out.) More seriously, Kövari and Pommerenke do not consider the fact that (2.6) is not valid for $n=0$. In this case we have

$$
\varphi_{0} \equiv 1=\frac{1}{2 \pi} \int_{0}^{2 \pi} d_{\theta} v\left(\theta, \theta_{0}\right) .
$$

Consequently, the proof requires a minor modification to deal with this case, with the result that the numerical interpretation of the bound given by Elliott also needs slight modification to the bounded part, although the dominant logarithmic term remains unchanged.

THEOREM 2.3. Let $D$ be a Jordan region whose boundary $\Gamma$ is of total rotation $V$ (with the usual modification if $D$ degenerates to an arc). We have

$$
\left\|F_{n}\right\| \leqslant \frac{V}{\pi}\left\{\frac{4}{\pi^{2}} \ln n+B\right\}, \quad n \geqslant 1,
$$

where $B$ is a certain absolute constant which (from numerical values computed in Geddes and Mason [12]) has the value 1.773 to 3 decimal places.

Proof. We recall that $\left\|F_{n}\right\|=\sup _{f \in A(D) ;\|f\|_{\infty}=1}\left\|F_{n}(f)\right\|_{\infty}$. Thus, let $f \in A(D)$, $\|f\|_{\infty}=1 .\left|F_{n}(f)(z)\right|, z \in D$, must, in view of the maximum principle, achieve its greatest value at some point $z_{0}=\psi\left(\rho e^{i \theta_{0}}\right) \in \Gamma$. Thus from (2.1)

$$
\begin{aligned}
\left\|F_{n}(f)\right\|_{\infty} & =\left|\sum_{j=0}^{n} \frac{1}{2 \pi i} \int_{|w|=\rho} \frac{f(\psi(w))}{w^{j+1}} d w \varphi_{j}\left(z_{0}\right)\right| \\
& \leqslant \frac{1}{2 \pi} \int_{|w|=\rho}\left|\sum_{j=0}^{n} \frac{\varphi_{j}\left(z_{0}\right)}{w^{j}}\right| \frac{d w}{w} \mid
\end{aligned}
$$

since $\|f\|_{\infty}=1$. Hence (2.6) and (2.7) yield

$$
\left\|F_{n}\right\| \leqslant \frac{1}{2 \pi} \int_{|w|=\rho \mid}\left|\sum_{j=0}^{n} \frac{1}{w^{j}} \frac{\rho^{j}}{\pi} \int_{0}^{2 \pi} e^{i j \theta} d_{\theta} v\left(\theta, \theta_{0}\right)\right|\left|\frac{d w}{w}\right|,
$$

where ' signifies that the first term in the sum is to be halved. Thus

$$
\left\|F_{n}\right\| \leqslant \frac{1}{2 \pi^{2}} \int_{0}^{2 \pi} \int_{|w|=\rho}\left|\sum_{j=0}^{n}\left(\frac{\rho e^{i \theta}}{w}\right)^{j}\right|\left|\frac{d w}{w}\right|\left|d_{\theta} v\left(\theta, \theta_{0}\right)\right| .
$$

The substitution $w=\rho e^{-i(t-\theta)}$ yields

$$
\int_{|w|=\rho}\left|\sum_{j=0}^{n}\left(\frac{\rho e^{i \theta}}{w}\right)^{j}\right|\left|\frac{d w}{w}\right|=\int_{0}^{2 \pi}\left|\sum_{j=0}^{n} e^{i j t}\right| d t=\int_{0}^{2 \pi}\left|\left(\sum_{j=0}^{n} e^{i j t}\right)-\frac{1}{2}\right| d t,
$$

whence from (2.2)

$$
\left\|F_{n}\right\| \leqslant \frac{V}{\pi}\left(\frac{1}{2}+\tau_{n}\right), \quad \text { where } \tau_{n}=\frac{1}{2 \pi} \int_{0}^{2 \pi}\left|\frac{e^{i(n+1) t}-1}{e^{i t}-1}\right| d t .
$$

The value of the integral $\tau_{n}$ has been extensively discussed by Geddes and Mason [12], and in particular (2.8) follows readily from their results.

Remark. In the case that $D$ is convex (so that $V=2 \pi$ ) we obtain immediately from (2.8) that $\left\|F_{n}\right\|<9$ for $n \leqslant 835$. 
3. Numerical Methods for Faber Series. Elliott [8], computes the Faber polynomials for certain regions directly from the definition, i.e. by obtaining the series for $\varphi$ and then using it to compute explicitly the series for $(\varphi)^{n}$. However, as it stands, this technique is not really suited to automatic computation, and we propose either the use of Fast Fourier Transform (FFT) methods or recursive techniques. The idea of using the FFT to evaluate Laurent coefficients appears to have been first proposed by Lyness and Sande [21]. FFT methods were also used by Geddes and Mason [12], and Geddes [13], to compute Maclaurin and Chebyshev coefficients. The use of recursive formulae to generate sequences of orthogonal polynomials on the real line is, of course, commonplace (see e.g. Fox and Parker [9]). The Faber series has many analogies with such orthogonal expansions.

We first propose two methods for the construction of the Faber polynomials themselves, and report on some numerical experiments. Provided $\psi$ is known, computation of the Faber coefficients for a given function $f$ is straightforward, and we defer this until later.

3.1. Computation of Faber Polynomials Using the FFT. If $\varphi_{n}(z)=\sum_{j=0}^{n} c_{j} z^{j}$, we have (2.3)

$$
c_{j}=\frac{1}{2 \pi i} \int_{|z|=R} \frac{\{\varphi(z)\}^{n}}{z^{j+1}} d z .
$$

Following Lyness and Sande [21], we first rewrite this as

$$
c_{j}=\frac{1}{2 \pi} \int_{0}^{2 \pi} \frac{\left\{\varphi\left(R e^{i \theta}\right)\right\}^{n}}{\left(R e^{i \theta}\right)^{j}} d \theta .
$$

Replacing this integral by its $N$-point trapezium rule approximation, we obtain

$$
c_{j} \approx \frac{1}{N R^{j}} \sum_{k=0}^{N-1}\left\{\varphi\left(R e^{i \theta_{k}}\right)\right\}^{n} e^{-j i \theta_{k}},
$$

where $\theta_{k}=2 \pi k / N$.

Thus all the coefficients $c_{j}, j=0, \ldots, n$, can be computed simultaneously using the FFT. (Since in fact we know that $c_{n}=1$, we have a useful check on the accuracy.) In view of the cheapness of this computation, one may as well take $N$ fairly large, and a suitable value on the CDC installation at ETH-Zentrum, Zürich, was found to be $N=512$. The IMSL subroutine FFT2C was used for the FFT. In practice it was found that some care has to be taken with the choice of $R$. If $R$ is too large, the values of $\left\{\varphi\left(R e^{i \theta_{k}}\right)\right\}^{n}$ lie relatively nearly on a circle, and accuracy is lost due to cancellation of figures for small $j$ and large $n$. On the other hand if $R$ is too small, so that the circle $|z|=R$ only just encloses $D$, singularities of $\varphi$ on the boundary $\Gamma$ may destroy the accuracy of the trapezium rule approximation. Somewhat surprisingly, we found that the former effect was much stronger than the latter, and a good rule for choosing $R$ has been found to be

$$
R=1.1 \times \max _{z \in \Gamma}|z| .
$$

We note in passing that it is often not convenient in practice to scale $\varphi$ so that $\lim _{z \rightarrow \infty} \varphi(z) / z=1$ (e.g. we may have the mapping onto the exterior of the unit circle). A simple modification of the program enables this scaling to be done 
automatically. If $\varphi$ is scaled so that the coefficient of $z$ in its expansion at $\infty$ is $\alpha$ instead of 1 , then the leading coefficient of $(\varphi)^{n}$ is $\alpha^{n}$, instead of 1 . If (3.1) is applied without rescaling $\varphi$, the value of $\alpha$ can be obtained from $c_{1}$ with $n=1$, and for each $n=1,2, \ldots$ the coefficients $c_{j}$ need only be divided by $\alpha^{n}$.

Several numerical examples have been computed to test the effectiveness of this method. (The values used for $N$ and $R$ are those given above, except where otherwise stated.)

Example 1. $D=\{z|| z+1 \mid \leqslant 1\}$. Thus $\varphi(z)=z+1$ and the coefficients of $\varphi_{n}$ are simply the $n$th binomial coefficients. Since $\varphi$ is entire, any value of $R$ may in theory be used, but in practice the lack of singularities appears to be a positive disadvantage, and, of all the examples tried, this proved to be the most dependent on $R$. With $R=1$, however, it was possible to obtain the coefficients to at least 10 significant figures for $n \leqslant 10$; about 9 figures for $n=20$ and about 6 figures for $n=31$, the largest value of $n$ tried.

Example 2. $D=[-1,1]$. In this case (see e.g. Markushevich [22]) the polynomials $\varphi_{n}$ are suitably normalized Chebyshev polynomials. For $n=12$ (the highest value of $n$ for which $T_{n}$ is given in Abramowitz and Stegun [1]) the coefficients obtained were virtually exact. For $n=30$, the correct value of $c_{0}$ is $-1 / 2^{29}=-1.8626 \cdots \times 10^{-9}$, and the program produced the value $-1.87117 \cdots \times 10^{-9}$.

Example 3. $D$ is the unit semidisc, i.e. $D=\{z|| z \mid \leqslant 1, \operatorname{Re}(z) \geqslant 0\}$. The mapping $\varphi$ is

$$
\varphi(z)=\rho\left(\frac{\beta(z-i)^{2 / 3}-\bar{\beta}(z+i)^{2 / 3}}{(z+i)^{2 / 3}-(z-i)^{2 / 3}}\right),
$$

where $\beta=e^{i \pi / 3}$. (Elliott [8] gives the value of $\rho$ as $4 /(3 \sqrt{3})$, but as pointed out above, using the FFT method, $\rho$ is not required explicitly.) The coefficients obtained for $\varphi_{n}$ agree to at least 7 figures with those obtained by Elliott for $n \leqslant 9$ (these are only given to 8 decimal places).

Example 4. We have used this method to calculate the Faber polynomials for the unit square and for various rectangles, using the Schwarz-Christoffel transformation which gives $\psi^{\prime}$. The main difficulty here is in actually obtaining the values of $\varphi$. We achieved this by using the IMSL automatic differential equation subroutine DGEAR to solve

$$
\frac{d z}{d w}=\frac{1}{\psi^{\prime}(w)}
$$

we will not give more details here since in practice it would appear to be better to use Method 2 (see below) for this problem. The results obtained using this method and that described below were in most cases virtually identical. However, for the unit square, $D=\{z|| \operatorname{Re}(z)|\leqslant 1,| \operatorname{Im}(z) \mid \leqslant 1\}$, and with $n=16$, some of the $c_{j}$ differed in the 8th decimal place from those given by Elliott. This slight discrepancy may be due to the fact that we used a more accurate value for the normalizing constant in the mapping (see below).

Before discussing the recursive method, we note that (2.4) provides a possible alternative formula for calculating the $c_{j}$ if $\psi$ is very much simpler than $\varphi$. However, we have performed no numerical experiments with this formula. 
3.2. Recursive Computation of Faber Polynomials. We recall (2.5)

$$
\varphi_{n+1}(z)=z \varphi_{n}(z)-\sum_{k=0}^{n-1} b_{k} \varphi_{n-k}(z)-(1+n) b_{n}, \quad n \geqslant 0,
$$

where $b_{k}$ is the coefficient of $w^{-k}$ in the expansion of $\psi$ at $\infty$. Since $\varphi_{0} \equiv 1$, this expression provides a recursive method of generating the Faber polynomials. Given $\psi$, it would, of course, be possible to generate the coefficients $b_{k}$ using the FFT, but in view of the possible dangers of instability or cancellation inherent in a recurrence, Method 1 would seem to be preferable in general. However, in the case when $D$ is a polygon, the Schwarz-Christoffel transformation can be used to obtain $\psi^{\prime}$, and the expansion of $\psi$ is then obtained rather easily. The mapping from the exterior of the unit circle onto the exterior of a polygon with exterior angles $\pi \xi_{i}, i=1,2, \ldots, m$, can be expressed as

$$
z=\gamma(u):=K \int\left(1-\frac{\eta_{1}}{u}\right)^{\xi_{1}-1}\left(1-\frac{\eta_{2}}{u}\right)^{\xi_{2}-1} \cdots\left(1-\frac{\eta_{m}}{u}\right)^{\xi_{m}-1} d u
$$

The ratio of the lengths of the sides is determined by the $\eta_{i}$ : sometimes the values required to obtain a given polygon can be calculated explicitly (e.g., by considerations of symmetry) but in general a numerical method must be used (see e.g., Trefethen [29]; Trefethen [30]). The position, size and orientation of the polygon are determined by $K$ and the constant of integration. We note that $\lim _{u \rightarrow \infty} z / u=K$. Thus substitution of $w=K u$ gives the required mapping $\psi$ and $\rho=|K|$. Expansion of each bracket, collection of powers of $1 / w$ and termwise integration gives the expansion for $\psi$. Details of the calculation in the general case can be found in Nepritvorennaja [23]. Here we treat the important special case of a rectangle since this permits the expansion to be considerably simplified. Further simplification occurs for a square and since this problem is considered by Elliott [8], we deal first with this case. It will be readily verified that for a square (3.2) becomes

$$
z=K \int\left(1+\frac{1}{u^{4}}\right)^{1 / 2} d u
$$

For the unit square $D=\{z|| \operatorname{Re}(z)|\leqslant 1,| \operatorname{Im}(z) \mid \leqslant 1\}$ Elliott gave the value of $K$ to 9 figures, obtained by applying the Euler transform to the series at $u=1$. This was not sufficiently accurate for our purposes, and using the numerical integration method described below for a general rectangle, we obtained the value $K=$ 1.1803405990161. Substituting $w=K u$ in (3.3) gives

$$
z=\int\left(1+\frac{K^{4}}{w^{4}}\right)^{1 / 2} d w=\int\left(1+\frac{K^{4}}{2 w^{4}}-\frac{K^{8}}{8 w^{8}}+\frac{K^{12}}{16 w^{12}} \cdots\right) d w .
$$

Integrating with respect to $w$ and noting that, in view of the symmetry of the unit square about the origin, the constant term must be zero, gives

$$
\psi(w)=w-\frac{K^{4}}{6 w^{3}}+\frac{K^{8}}{56 w^{7}}-\frac{K^{12}}{176 w^{11}}+\cdots
$$

whence by definition

$$
b_{0}=b_{1}=b_{2}=0, \quad b_{3}=-K^{4} / 6, \quad b_{4}=b_{5}=b_{6}=0, \quad b_{7}=K^{8} / 56, \text { etc. }
$$


The recurrence (2.5) then yields immediately

$$
\begin{aligned}
& \varphi_{0}(z)=1, \quad \varphi_{1}(z)=z, \quad \varphi_{2}(z)=z^{2}, \quad \varphi_{3}(z)=z^{3}, \\
& \varphi_{4}(z)=z^{4}+2 K^{4} / 3, \quad \varphi_{5}(z)=z^{5}+\left(5 K^{4} / 6\right) z, \text { etc. }
\end{aligned}
$$

It will be seen that this calculation is very much simpler than that used by Elliott for this problem, which, following the method described by Nepritvorennaja, involves determining the power series for $\varphi$.

We turn now to the case of the rectangle

$$
D=\{z|| \operatorname{Re}(z)|\leqslant A,| \operatorname{Im}(z) \mid \leqslant B\},
$$

for which (3.2) becomes

$$
z=\gamma(u)=K \int\left(1+\frac{C}{u^{2}}+\frac{1}{u^{4}}\right)^{1 / 2} d u, \quad-2<C<2 .
$$

We must first determine the constants $K$ and $C$. Note that by symmetry, the points 1 and $i$ on the unit circle are mapped to $A$ and $i B$, respectively. Thus if

$$
I(C):=\int_{1}^{i}\left(1+\frac{C}{u^{2}}+\frac{1}{u^{4}}\right) d u,
$$

where the path of integration satisfies $|u| \geqslant 1$,

$$
\frac{A}{B}=\frac{\operatorname{Re}(-I(C))}{\operatorname{Im}(I(C))}
$$

and the required value of $C$ can easily be determined using the secant rule in terms of the variable $t$ defined by $C=2\left(1-t^{2}\right) /\left(1+t^{2}\right), 0<t<\infty$. In practice we chose the path of integration to be the circular arc

$$
u=\frac{(1+i)}{2}+\frac{e^{i \theta}}{2}, \quad \frac{-\pi}{4} \leqslant \theta \leqslant \frac{3 \pi}{4}
$$

and the integration was performed using the IMSL automatic quadrature subroutine DCADRE. $K$ is then determined from the known values of the mapping at 1 and $i$ on the unit circle, and the value of $I(C)$. We now proceed as described above by substituting $w=K u$, and expanding to obtain

$$
\psi^{\prime}(w)=\sum_{j=0}^{\infty}\left(\begin{array}{c}
1 / 2 \\
j
\end{array}\right) \sum_{k=0}^{j}\left(\begin{array}{l}
j \\
k
\end{array}\right)\left(\frac{K^{2} C}{w^{2}}\right)^{k}\left(\frac{K^{4}}{w^{4}}\right)^{j-k} .
$$

(Here and below, $C^{0}$ is interpreted as 1 if $C=0$.) Collecting powers of $1 / w$, followed by termwise integration (again making use of the fact that by symmetry the constant term in the expansion of $\psi$ is zero), yields $b_{j}=0, j$ even, and

$$
b_{2 k-1}=\frac{K^{2 k}}{1-2 k} \sum_{m=[(k+1) / 2]}^{k} \frac{(1 / 2)^{(m)} C^{2 m-k}}{(2 m-k) !(k-m) !}, \quad k=1,2, \ldots,
$$

where [ ] denotes the integer part and

$$
(1 / 2)^{(m)}:=\frac{1}{2}\left(\frac{1}{2}-1\right)\left(\frac{1}{2}-2\right) \cdots\left(\frac{1}{2}-m+1\right) .
$$

Since the $b_{j}$ 's are real, there is no serious inconvenience in computing them and the recurrence (2.5) to double precision. 
With this precaution, no sign of instability was observed, and the method proved both effective and efficient.

3.3. Computation of the Faber Coefficients. We have (2.1) for the Faber coefficients for $f \in \hat{A}(D)$ :

$$
a_{n}=\frac{1}{2 \pi i} \int_{|w|=R} \frac{f(\psi(w))}{w^{n+1}} d w .
$$

We may simply replace the integral by its $N$-point trapezium rule approximation and evaluate as many of the $a_{n}$ as we require using the FFT (compare Geddes [13]). In general the choice of $R$ in this formula does not appear to be critical. If $\Gamma$ is a smooth curve, one might as well choose $R=\rho$, but, if not, $R$ should be chosen slightly greater than $\rho$ (e.g., $1 \cdot 1 \rho$ ) to avoid a singular integrand. The only problem is ensuring that the level curve $\Gamma_{R}$ does not enclose or pass too close to a singularity of $f$ : generally this is not immediately obvious. The simplest way out of this difficulty is to guess a suitable value of $R$, plot or print out points on $\Gamma_{R}$ and then check its position in relation to the singularities of $f$. On the examples tried, we have not found any difficulty in practice in choosing $R$, and we have successfully produced expansions of several functions on the semidisc; ellipses (the case considered by Geddes [13]); the unit square and various rectangles. For the semidisc $D=\{z|| z \mid \leqslant$ $1, \operatorname{Re}(z) \geqslant 0\}$, the errors can in some cases be compared with some best approximation errors $E_{n}$ computed by Elliott [8]. For instance, we have $\left\|e^{z}-F_{4}\left(e^{z}\right)\right\|_{\infty}=.45$ $\times 10^{-2}$ compared with $E_{4}=.38 \times 10^{-2} ;\left\|e^{z}-F_{6}\left(e^{z}\right)\right\|_{\infty}=.65 \times 10^{-4}$ compared with $E_{6}=.51 \times 10^{-4}$. For this example, the computed values of $\left\|e^{z}-F_{n}\left(e^{z}\right)\right\|_{\infty}$ decreased smoothly with $n$ down to a value of $.23 \times 10^{-11}$ at $n=13$, after which no significant improvement occurred: the smallest value obtained was $\left\|e^{z}-F_{14}\left(e^{z}\right)\right\|_{\infty}$ $=.19 \times 10^{-11}$. (These smallest relative values of around $10^{-11}$ to $10^{-12}$ were fairly typical of those that could be achieved on the CDC machine with the values of the parameters discussed above.) With $f(z)=(1+2 z)^{-1 / 2}$, we obtained $\left\|f-F_{4}(f)\right\|_{\infty}$ $=.64 \times 10^{-1}\left(E_{4}=.42 \times 10^{-1}\right)$, and $\left\|f-F_{6}(f)\right\|_{\infty}=.26 \times 10^{-1}\left(E_{6}=.17 \times\right.$ $\left.10^{-1}\right)$. Convergence for this example is quite slow due to the singularity of $f$ at $z=-\frac{1}{2}$ (compare Corollary 2.2(b)), and the errors obtained decreased smoothly down to $\left\|f-F_{31}(f)\right\|_{\infty}=.12 \times 10^{-5}$, the largest value of $n$ tried.

It will be noted that a difficulty arises in evaluating $\psi(w)$ when the SchwarzChristoffel transformation is used. However since values are required only around the circle $w=R e^{i \theta}, 0 \leqslant \theta<2 \pi$, we can once again use the FFT to sum the series for $\psi$ at all the required points simultaneously. In order to do this one requires rather a lot of values of $b_{k}$ (we used 512 terms). These could be computed by the method discussed above but for this purpose we do not require quite such high accuracy, particularly for large $k$, so it is preferable to compute the Laurent coefficients of $\psi^{\prime}$ using the FFT and then get the $b_{k}$ by termwise integration: in this way evaluation of $\psi$ can be made quite efficient.

Finally we consider the computation of expansions for $f \in A(D)-\hat{A}(D)$. In this case the degree of polynomial approximation is unlikely to be sufficiently good to make polynomial approximation an attractive proposition. Nevertheless Theorem 2.3 remains valid, and the expansion will still produce polynomial approximations very close to the best one (for reasonable values of $n$ ) should these be required for 
some purpose. In this case one is obliged to take $R=\rho$ in (2.3) (i.e., integrate round $\Gamma$ itself) and the integrand is (by definition) singular, so rather than use the FFT method described above, it would be better to compute the coefficients $a_{n}$ individually using an adaptive quadrature program.

3.4. Evaluation of the Faber Series. Orthogonal polynomials on intervals of the real line satisfy a three term recurrence which can be used to set up a backward recurrence to evaluate the orthogonal expansion. (See e.g. Fox and Parker $[9$, p. 56].) In principle, (2.5) can be used in the same way, but if all the $b_{k}$ are nonzero this would be very expensive computationally since the recurrence in this case is of infinite order, so in general it is better to express the truncated series $F_{n}(f)$ in terms of powers of $z$ for the actual evaluation. However for regions such as the unit square, where only every fourth $b_{k}$ is nonzero, direct evaluation using (2.5) may be useful.

3.5. Using Approximate or Numerical Conformal Mapping. For a general region $D$, the mapping $\varphi$ may not be known and numerical conformal mapping must be used. See Gaier [10], for a general survey of methods up to that date. As discussed below, methods yielding analytic approximations to $\varphi$ or $\psi$, rather than integral equation methods, are more appropriate here. For recent work on such methods, see Levin, Papamichael and Sideridis [20]; Ellacott [7]; Hoidn [16]. (This last paper actually describes the author's method for doubly connected regions, but also contains improvements in the technique over the original description in Grassmann [15], of the method for the simply connected case.) These methods are all described for the problem of mapping the region inside a Jordan curve onto the interior of the disc: To obtain our mapping $\varphi$ an initial inversion in an interior point of $D$ and a final inversion in the origin must be performed. Unfortunately, however, unless the region $D$ is fairly simple, the methods are not sufficiently good to get the $\varphi_{n}$ 's accurately, and we must adopt a slightly different point of view. The conformal mapping method must be regarded as producing a mapping $\tilde{\varphi}$ which maps the complement of a region $\tilde{D} \supset D$ onto $|w|>\hat{\rho} \geqslant \rho$. Most conformal mapping algorithms based on approximations have the property of giving mappings which are eventually conformal (for an approximation of sufficiently high order) on compact subsets not intersecting the boundary, so if an approximation is produced for which the deviation from constant modulus of the image of $\Gamma$ is reasonably small (say about $1 \%$ ) we will not require to take $\tilde{\rho}$ very much larger than $\rho$ for $\tilde{\varphi}$ to be conformal, and hence $\tilde{D}$ will be a good fit around $D$. A Faber expansion of our given function $f$ on $\tilde{D}$ should thus provide a good approximation to $f$ on $D$. One may identify three desirable properties for the mapping algorithm.

(i) The method should reliably generate a reasonably good approximation even for difficult regions. (On the other hand, very high accuracy is not required.)

(ii) The inverse of the approximate mapping should be readily available.

(iii) In view of Theorem 2.3, one would like to have some form of variation diminishing property on the boundary $\Gamma$.

We are aware of no method satisfying property (iii) (but see below); on the contrary most methods tend to introduce boundary oscillations. On the other hand (i) and (ii) are satisfied by the Hoidn-Grassmann method and this would seem to be the most hopeful; the relatively poor asymptotic properties of the method are not significant here. With an initial inversion in the point 1.5, 20 iterations of the 
Hoidn-Grassmann method, and a final inversion in the origin applied to the "semiannulus"

$$
D=\{z|1 \leqslant| z \mid \leqslant 2, \operatorname{Re}(z) \geqslant 0\},
$$

we have computed expansions of $e^{z}$ and $\ln z$. For $e^{z}$, we were able to obtain an approximation with an error (on the exact region $D$ ) of $1.4 \times 10^{-10}$. Convergence for $\ln z$ is quite slow: evaluation of the approximate mapping at $z=0$ indicates that this point lies on a level curve for which $R / \rho$ (see Corollary 2.2(b)) is only about 1.17. With $n=31$, an approximation with error (on $D$ ) of $.14 \times 10^{-2}$ was achieved.

A disadvantage with the Hoidn-Grassmann method is that symmetry about the real axis is not preserved. Thus the resulting approximations may have complex coefficients. However, it is easily verified that if $f$ and $D$ are symmetric about the real axis (i.e. $z \in D \rightarrow \bar{z} \in D, f(\bar{z})=\overline{f(z)}$ ), then for any polynomial $p=\sum_{j=0}^{n} a_{j} z^{j}$,

$$
\left\|f-\sum_{j=0}^{n} \operatorname{Re}\left(a_{j}\right) z^{j}\right\|_{\infty} \leqslant\|f-p\|_{\infty} .
$$

Before leaving this problem we note that a possible alternative approach to obtaining $D$ is to interpolate or otherwise approximate $\Gamma$ by a polygon. This method would satisfy property (iii) but would present other problems in determining the coefficients of the Schwarz-Christoffel mapping etc.

4. Concluding Remarks. It has been found that the Faber series provides an effective and efficient method for producing near best approximations to analytic functions in the complex plane, especially when the mapping $\varphi$ for the required domain $D$ is available. However, the method can still be used even if $\varphi$ is not known explicitly.

Forschungsinstitut für Mathematik

ETH

Zürich, Switzerland

Department of Mathematics

Brighton Polytechnic

Brighton BN2 4GJ, England

1. M. Abramowitz \& I. A. Stegun, Handbook of Mathematical Functions (9th Dover Printing based on the U. S. Government Printing Office edition), Dover, New York, 1972.

2. I. Barrodale, L. M. Delves \& J. C. Mason, "Linear Chebyshev approximation of complex-valued functions," Math. Comp., v. 32, 1978, pp. 853-863.

3. H.-P. Blatt, "A general Remes algorithm in real or complex normed linear spaces," Multivariate Approximation (D. Handscomb, ed.), Academic Press, London, 1978, pp. 145-153.

4. E. W. Cheney \& K. H. Price, "Minimal projections," in Approximation Theory (A. Talbot, ed.), Academic Press, London, 1970, pp. 261-289.

5. J. H. CURTiss, "Faber polynomials and the Faber series," Amer. Math. Monthly, v. 78, 1971, pp. $577-596$.

6. S. W. Ellacott \& J. Williams, "Linear Chebyshev approximation in the complex plane using Lawson's algorithm,” Math. Comp., v. 30, 1976, pp.35-44.

7. S. W. Ellacort, A technique for approximate conformal mapping," Multivariate Approximation (D. Handscomb, ed.), Academic Press, London, 1978, pp. 301-314.

8. G. H. Elliotr, The Construction of Chebyshev Approximations in the Complex Plane, Ph.D. Thesis, Faculty of Science (Mathematics), University of London, 1978. 1968.

9. L. Fox \& I. B. PARKer, Chebyshev Polynomials in Numerical Analysis, Oxford Univ. Press, London, 
10. D. GAIER, Konstruktive Methoden der konformen Abbildung, Springer-Verlag, Berlin, 1964.

11. D. GAIER, Vorlesungen über Approximation im Komplexen, Birkhäuser-Verlag, Basel, 1980.

12. K. O. Geddes \& J. C. Mason, "Polynomial approximation by projections on the unit circle," SIAM J. Numer. Anal., v. 12, 1975, pp. 111-120.

13. K. O. GEDDES, “Near minimax polynomial approximation in an elliptical region," SIAM J. Numer. Anal., v. 15, 1978, pp. 1225-1233.

14. K. GlashofF \& K. RolefF, "A new method for Chebyshev approximation of complex-valued functions," Math. Comp., v. 36, 1981, pp. 233-239.

15. E. GrassmanN, "Numerical experiments with a method of successive approximation for conformal mapping," Z. Angew. Math. Phys., v. 30, 1979, pp. 873-884.

16. H. P. HoIDN, "Osculation methods for the conformal mapping of doubly connected regions," $Z$. Angew. Math. Phys., v. 33, 1982.

17. M. H. GuTKNECHT, "Ein Abstiegsverfahren für nicht-diskrete Tschebysheff Approximationsprobleme," in Numerische Methoden der Approximationstheorie, Vol. 4 (L. Collatz, G. Meinardus and H. Werner, eds.), Birkhäuser-Verlag, Basel, 1978, pp. 154-171.

18. M. H. GutKnecht \& L. N. TRefethen, "Real polynomial Chebyshev approximation by the Carathéodory-Fejér method,” SIAM J. Numer. Anal., v. 19, 1982, pp. 358-371.

19. T. KOVARi \& CH. Pommerenke, “On Faber polynomials and Faber expansions," Math. Z., v. 99, 1967, pp. 193-206.

20. D. Levin, N. Papamichael \& A. Sideridis, "The Bergman kernel method for the numerical conformal mapping of simply connected domains," J. Inst. Math. Appl., v. 22, 1978, pp. 171-187.

21. J. N. LyNESS \& G. SANDE, "Algorithm 413: Evaluation of normalised Taylor coefficients of an analytic function," Comm. ACM, v. 14, 1971, pp. 669-675.

22. A. I. Markushevich, Theory of Functions of a Complex Variable, Translated and edited by R. A. Silverman (three volumes in one), Chelsea, New York, 1977.

23. L. M. Nepritvorennaja, "Construction of Faber polynomials for an arbitrary polygon," Bul. Akad. Śtiince RSS Moldoven., no. 3, 1973, pp. 80-83.

24. J. C. MASON, "Orthogonal polynomial approximation methods in numerical analysis," in Approximation Theory (A. Talbot, ed.), Academic Press, London, 1970, pp. 7-34.

25. G. OPFER, “An algorithm for the construction of best approximations based on Kolmogorov's criterion,” J. Approx. Theory, v. 23, 1978, pp. 299-317.

25. G. OPFER, "An algorithm for the construction of best approximations based on Kolmogorov's criterion,” J. Approx. Theory, v. 23, 1978, pp. 299-317.

26. Сh. Pommerenke, “Konforme Abbildung und Fekete-Punkte,” Math. Z., v. 89, 1965, pp. 422-438.

27. J. RADON, “Über die Randwertaufgaben beim logarithmischen Potential," Sitz.-Ber. Akad. Wiss., Wien. Math.-naturw. Kl., Abt. IIa, v. 128, 1919, pp. 1123-1167.

28. R. L. Streit \& A. H. Nutall, Linear Chebyshev Complex Function Approximation, NUSC report 6403, Naval Underwater Systems Center, New London, Conn., 1981.

29. L. N. TRefethen, "Numerical computation of the Schwarz-Christoffel transformation," SIAM J. Sci. Statist. Comput., v. 1, 1980, pp. 82-102.

30. L. N. Trefethen, "Computer application of the Schwarz-Christoffel transformation," in E. B. Christoffel-The Influence of His Work on Mathematics and the Physical Sciences (P. L. Butzer and F. Fehér, eds.), Birkhäuser, Basel, 1981, pp. 263-274.

31. L. N. Trefethen, "Near circularity of the error curve in complex Chebyshev approximation," $J$. Approx. Theory, v. 31, 1981, pp. 344-366. 